\title{
Método de Regularización de Mallas Cuadrilaterales en Reconstrucción de Objetos 3D
}

\author{
Sandra P. Mateus \\ Institución Universitaria de Envigado, Facultad de Ingeniería, Línea de Investigación en Inteligencia \\ Computacional, Carrera 27B No 39A Sur 57, Of. 201C, Envigado, Antioquia-Colombia \\ (e-mail: spmateus@iue.edu.co)
}

\begin{abstract}
Resumen
Se propone un método de regularización de una malla cuadrilateral mediante Geodésicas y BSplines aplicado a la reconstrucción de objetos 3D. El procedimiento realizado, se resume en tres etapas principales: i) selección de cuadriláteros; ii) regularización de los cuadriláteros y generación de puntos, utilizando B-Splines; y iii) emparejamiento de puntos regularizados mediante geodésicas con el método de la marcha rápida (fast marching method, FMM). En el proceso de experimentación, la regularización de la malla cuadrilateral y la representación computacional de los modelos se hicieron con una imagen de rango del objeto cultural moai. A pesar de que el objeto tiene topología arbitraria irregular, el método propuesto dio resultados adecuados en la conservación de los detalles finos del objeto.
\end{abstract}

Palabras clave: objetos 3D, malla cuadrilateral, B-Spline, geodésica, método de regularización

\section{Regularization Method of Quadrilaterals Mesh for 3D Object Reconstruction}

\begin{abstract}
A regularization method of a quadrilateral mesh by means Geodesics and B-Splines, applied to 3D objects reconstruction, is proposed. The procedure can be summarized in three main steps: i) selection of quadrilaterals; ii) regularization of quadrilaterals and generation of points using BSplines; and iii) matching regularized points by means of Fast Marching Method geodesic (FMM). In the process of experimentation, the regularization of the representation of the quadrilateral mesh and the representation of the computational models were done with a range image of the cultural object moai. Despite having an irregular arbitrary topology, the proposed method gave adequate results in the conservation of the fine detail of the object.
\end{abstract}

Keywords: 3D objects, quadrilateral mesh, B-Spline, geodesic, regularization method 


\section{INTRODUCCIÓN}

Reconstruir un objeto tridimensional, se refiere a obtener su representación en la memoria de un computador, manteniendo sus características de volumen y forma, partiendo de un conjunto discreto de puntos muestreados a partir de su superficie. La reconstrucción tridimensional es una tarea no trivial que en general involucra cinco etapas tales como: adquisición, registro, integración, segmentación y ajuste, siendo esta última la que proporciona el modelo computacional del objeto representado (Yvart et al., 2005). Una de las fases más importantes del proceso de reconstrucción 3D es el ajuste de superficies, dado que es en ésta, donde finalmente se obtiene el modelo matemático o representación computacional del objeto real. Existen muchos métodos que se pueden emplear para modelar superficies de objetos 3D, el ajuste por mallas triangulares (Bremer et al., 2004) y por superficies NURBS (Non-Uniform Rational B-Splines), son algunos de ellos.

Las mallas triangulares con frecuencia exhiben varias deficiencias. Ellas son frecuentemente parametrizadas con resoluciones inapropiadas y típicamente contienen muchos elementos formados de manera inadecuada. Debido a la constante ocurrencia de mallas deficientes, la habilidad de segmentar las superficies en regiones formadas adecuadamente y de reparametrizar superficies con mallas bien formadas, es de importancia crítica en casi todos los sistemas de procesamiento de mallas. Es por lo anterior, que se hace necesario reparametrizar estas mallas para un modelamiento adecuado. La reparametrización de las mallas triangulares es uno de los procesos fundamentales usados por casi todos los sistemas de procesamiento geométrico. La mayoría de trabajos se han enfocado en el remallado triangular; el problema igualmente importante de la reparametrización de superficies trianguladas en cuadriláteros ha permanecido por mucho tiempo sin dirección (Ni et al., 2004). A pesar de la falta relativa de atención, la necesidad de métodos de reparametrización cuadrilaterales de calidad es de gran importancia en varias áreas de computación gráfica y visión por computador. Los cuadriláteros son las primitivas preferidas para el modelamiento de muchos objetos y en muchos dominios de simulación; muchas formulaciones de subdivisión de superficies como los Splines y las NURBS, requieren bases cuadrilaterales complejas; descomponer una superficie en cuadriláteros bien formados provee un medio casi natural para la construcción de parches NURBS (Dong et al., 2005). Sin embargo, sólo recientemente han sido desarrollado métodos para cuadrilaterizar automáticamente superficies complejas; esos pocos métodos que existen son bastante complejos, difíciles de implementar y cuentan con múltiples componentes heurísticos, haciéndolos impropios para usarlos en determinados dominios de la computación gráfica y de la visión por computador, de manera general.

Una malla dada representa un muestreo organizado de algunas superficies fundamentales, descompuestas en una colección arreglada de polígonos. Sin embargo, este muestreo no provee generalmente, un ajuste deseable para todas las aplicaciones. Consecuentemente, el reparticionamiento y el remuestreo de una malla son herramientas necesarias en los sistemas de procesamiento de mallas. Esto está involucrado en un número considerable de problemas, que han sido extensamente estudiados. A continuación se revisan los más relevantes.

\section{Simplificación de la Malla}

Las mallas pueden ser construidas desde nubes de puntos, datos de rango y de extracción de isosuperficies. La forma objetivo, puede casi siempre ser representada muy bien usando unos polígonos distantes entre sí. Muchos métodos de simplificación de superficies han sido desarrollados para derivar tales aproximaciones simples automáticamente (Garland et al., 2001). La mayoría de algoritmos de simplificación operan aplicando una primitiva de mortandad, tal como la contracción de bordes, o levantamiento de vértices (Schroeder et al., 1992). Una aproximación más general es la aplicación operativa de operaciones, tales como la contracción de bordes sin una búsqueda de optimización global (Hoppe, 1996). Estos métodos se enfocan en el adaptamiento de la conectividad de la malla. Un énfasis similar en dirección al remuestreo de la superficie puede ser visto en métodos que construyen una descomposición global de la superficie en parches, los cuales son poligonizados para producir una malla final (Cohen-Steiner et al., 2004). 


\section{Remallado semi-regular}

El objetivo de los trabajos en esta área es reducir la complejidad de la malla, mientras se mantiene la fidelidad geométrica; las medidas de calidad tal como el grado de los vértices y la regularidad de la malla son generalmente ignoradas. Para un patrón de métodos de análisis de malla multiresolución, el máximo interés está, en cambio, en que la malla tenga la mejor regularidad posible. Así la estructura de la malla regular es importante, en parte, porque esta puede ser derivada por operaciones de subdivisión de mallas recursivas.

Los esquemas de remallado semiregular aproximan este problema, a un proceso de dos pasos. Primero ellos descomponen la superficie en un conjunto de triángulos o parches cuadrilaterales. Segundo, ellos remuestrean cada parche por subdivisión recursiva. De forma similar, los sistemas MAPS (Koren et al., 2002) construyen un dominio de base triangular por simplificación. Ambas son diseñadas para usarlas como la etapa inicial en análisis de subdivisión de superficies. Mas recientemente, esta aproximación fundamental ha sido extendida por construcción de mallas normales (Friedel et al., 2004), los cuales se enfocan primariamente en la eficiencia codificada de la jerarquía multiresolución y en la parametrización suave global (Karni y Gotsman, 2000), las cuales producen un alto grado de suavidad paramétrica alrededor de los límites de parches triangulares. En contraste al caso triangular, comparativamente pocos trabajos se han direccionado en el problema de construir mallas cuadrilaterales semi-regulares. La aproximación más común ha tomado esta para construir una descomposición no-cuadrilateral, de la cual una cuadrilaterización ha sido derivada. Eck y Hoppe (1996), inician construyendo una base triangular compleja. Ellos resuelven un problema de emparejamiento máximo sobre el grafo dual de este complejo, uniendo todos los triángulos pares en cuadriláteros. Similarmente, Boier-Martin et al. (2004) producen un agrupamiento arbitrario de la superficie en conjuntos de caras conectadas, los cuales los límites deben ser polígonos de algún tipo y entonces cuadrilaterizan estos polígonos.

Recientemente, Solín y Segeth (2007) proponen una nueva familia de elementos Hermite de orden superior jerárquicos en híbridos de mallas triangulares/cuadrilaterales. En este método, los modos de orden superior interiores, se calculan utilizando un valor propio (eigen-value) del problema generalizado, preservando la continuidad de las derivadas que se desarrollan en la red de vértices. Matthies y Schieweck (2007) estudian las propiedades de un mapeo referencial para cuadriláteros finitos, considerando rejillas adaptativas multinivel con nodos colgantes que normalmente son generados por refinamiento adaptativo desde una malla robusta regular. En la aplicación realizan pruebas de estimación con interpolación de error para mapeados discontinuos en cuadriláteros.

En este artículo, se presenta una metodología para regularizar una malla cuadrilateral generada previamente en objetos 3D. La malla cuadrilateral obtenida previamente, se regulariza mediante el uso de curvas geodésicas y B-Splines para obtener una rejilla óptima sobre la cual posteriormente sea sencillo trazar parches NURBS.

\section{METODOLOGÍA DE REGULARIZACIÓN DE LA MALLA CUADRILATERAL}

Es necesario regularizar los cuadriláteros obtenidos de una malla cuadrilateral para posteriormente ajustar la superficie por parches NURBS, ya que no todos los cuadriláteros de la malla pueden ser aptos para trazar NURBS directamente sobre ellos. De manera general, la metodología utilizada para regularizar estos parches se puede observar en la Figura 1.

La metodología mostrada en la Figura 1, hace énfasis en un cuadrilátero, pero esta fue aplicada a todos los cuadriláteros de la malla dada. En la Fig. 2, se puede observar como se selecciona primero uno de los cuadriláteros de la malla y posteriormente se selecciona un lado de tal cuadrilátero y su opuesto. El lado seleccionado, es aleatorio y para buscar su opuesto, se tiene en cuenta que los vértices de éste último lado, no los contenga el primer lado seleccionado; es decir que si el primer lado seleccionado del cuadrilátero tiene vértices $A$ y $B$, se garantiza que el otro lado no contenga ni a $A$ ni a $B$, por lo que resultaría $C$ y $D$ y así se garantiza que sea su opuesto. 


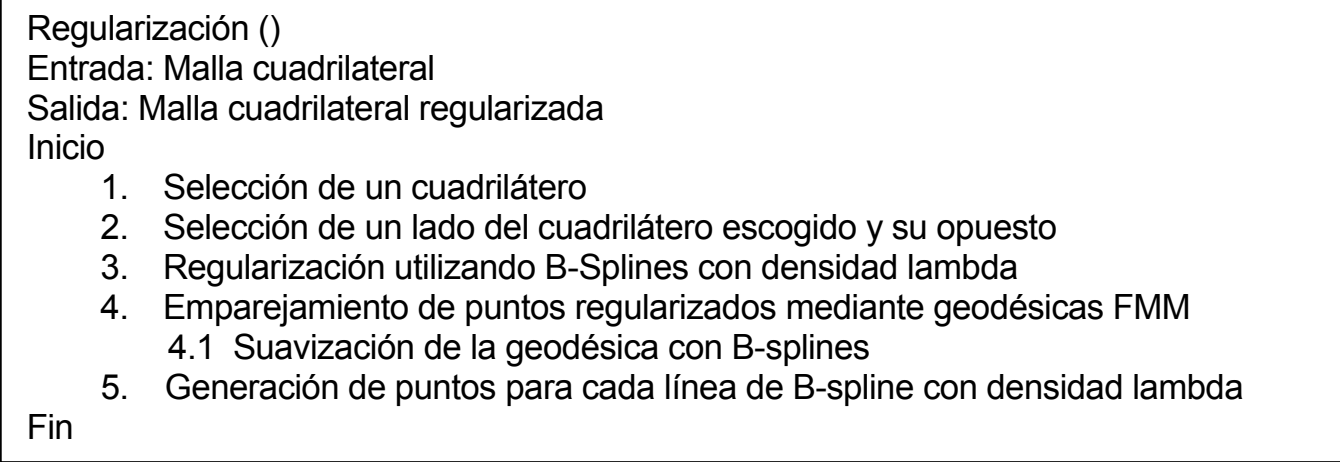

Fig. 1. Metodología utilizada para la regularización de la malla cuadrilateral

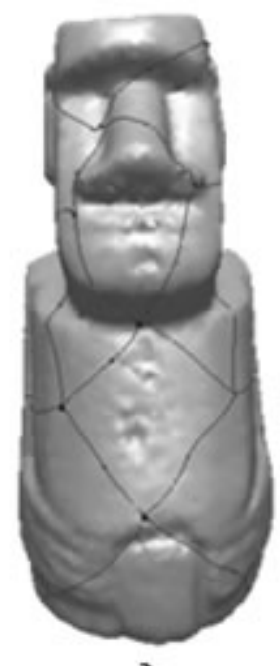

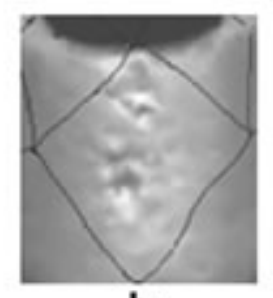

b.

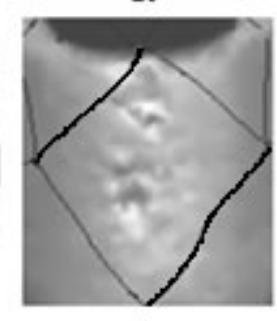

c.

Fig. 2. a. Malla Cuadrilateral. b. Selección del cuadrilátero. c. Selección de bordes opuestos del cuadrilátero seleccionado

Posterior al paso anterior, se trazaron B-splines sobre los lados seleccionados con una densidad $\lambda$ (Ver Fig. 3), para garantizar que se escogieran los mismos puntos para ambos lados, sin importar la distancia entre ellos; esto con el fin de tener una rejilla sobre la que se pueda trazar después la NURBS.

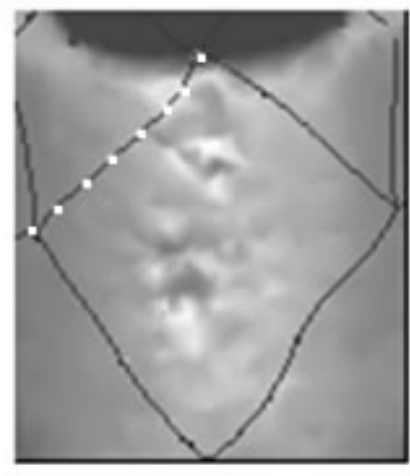

a.

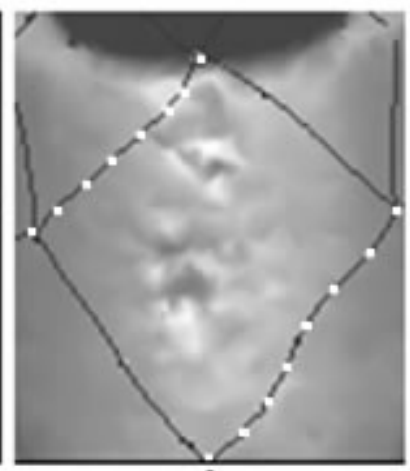

b.

Fig. 3. Regularización utilizando B-Splines

En forma general, una B-spline, no interpola todos sus puntos de control por lo que son curvas de aproximación que permiten una manipulación local de la curva y requieren menos cálculos para la determinación de sus coeficientes. Las curvas Spline son segmentos polinomiales donde el cambio de polinomios ocurre en puntos de control. Siendo $P(t)$ el vector posición alrededor de la curva que es función del parámetro t, una curva B-Spline está dada por la ecuación (1):

$P(t)=\sum_{i=1}^{n+1} B_{i} N_{i, k}(t) \quad t_{\min } \leq t \leq t_{\max }, 2 \leq k \leq n+1$ 
donde $B_{i}$ son los vectores posición de los $n+1$ puntos de control y los $N_{i, k}$ son las funciones base $B-$ Spline normalizadas.

Teniendo los puntos sobre los lados seleccionados, éstos se emparejaron. Para ello, primero se usó el algoritmo Dijkstra Continuo; en la Fig. 4a, se observa que este algoritmo produce un camino que a pesar de ser mínimo no es óptimo, ya que no va directo de punto a punto; éste algoritmo encuentra el camino más corto entre el origen y un punto especificado, produciendo un orden de magnitud de $\mathrm{O}\left(\mathrm{n} \log ^{2} \mathrm{n}\right)$, siendo $\mathrm{n}$ el número de vértices de la malla (Taubin, 2000). Por lo anterior, se exploró el FMM (Fast Marching Method) (Ver Fig. 4b); este algoritmo es usado para definir una función de distancia desde un punto de origen al resto de la superficie en un orden de magnitud de $O(n \log n)$, e integra una ecuación diferencial para obtener el camino geodésico más corto, recorriendo los vértices de los triángulos.

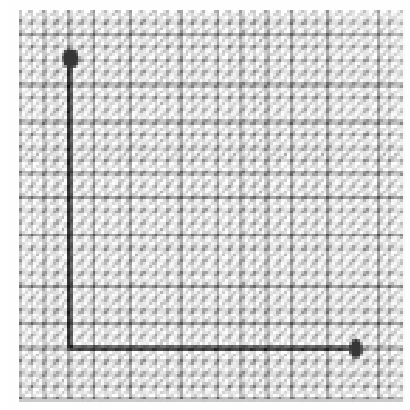

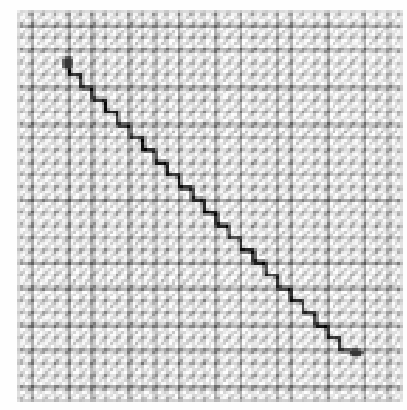

b.

Fig. 4. Emparejamiento de puntos por: a. Algoritmo Dijkstra Continuo. b. Algoritmo FMM

A pesar de con el algoritmo FMM se encontró un camino óptimo, fue necesario aplicar nuevamente BSplines para suavizar la curva creada por el algoritmo FMM, como se observa en la Fig. 5.

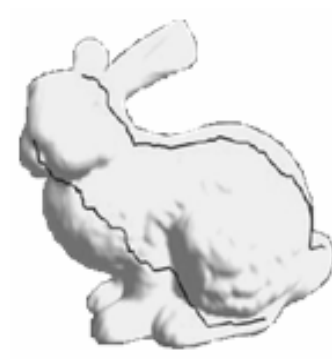

a.

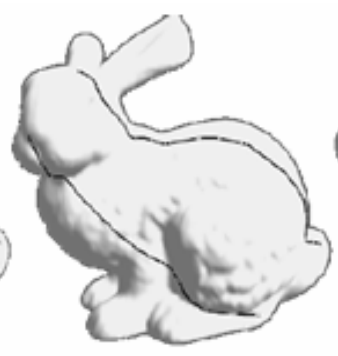

b.

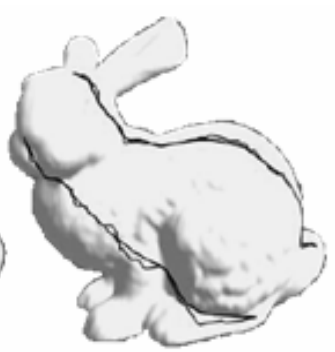

c.

Fig. 5. Emparejamiento de Puntos. a. Curva Geodésica FMM. b. Suavización de la Geodésica con BSpline. c. Curva Geodésica y B-Spline

Finalmente, para terminar de regularizar los cuadriláteros, al trazar los B-Splines para suavizar la geodésica, se volvieron a encontrar puntos con densidad $\lambda$ en cada una de las líneas que emparejaron los puntos iniciales (Ver Fig. 6), para obtener finalmente la rejilla que sirve para trazar el parche NURBS.

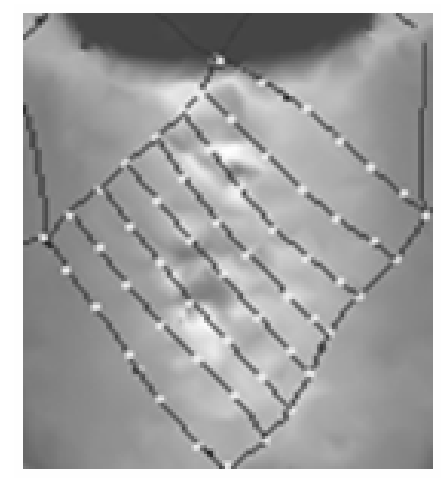

Fig. 6. Generación de puntos con densidad $\lambda$ en cada una de las curvas que emparejaron los puntos 


\section{RESULTADOS Y DISCUSIÓN}

Todas las pruebas fueron realizadas utilizando una computadora con procesador de AMD Semprom a $3,0 \mathrm{GHz}$, memoria RAM de $512 \mathrm{MB}$, corriendo bajo el sistema operativo Windows XP. Las implementaciones de los modelos fueron realizados en $\mathrm{C}++$ y Matlab, y se programó un motor gráfico en OpenGL, para obtener la representación gráfica de las imágenes.

En el proceso de experimentación, la generación de la malla cuadrilateral y la representación computacional de los modelos, fueron probados con una imagen de rango del objeto cultural moai del Max Planck Institut Informatik, el cual tiene topología arbitraria irregular. En las Fig. 7 y 8 , se puede observar la malla cuadrilateral obtenida previamente del objeto.

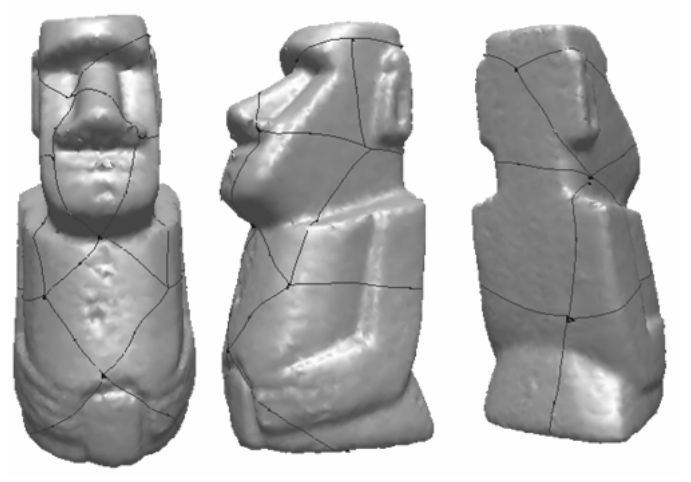

Fig. 7. Cuadrilaterización previa del Objeto Moai

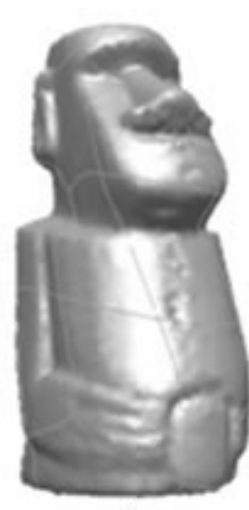

a.

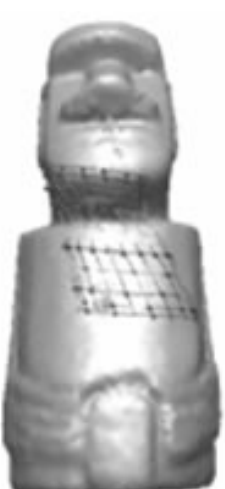

b.

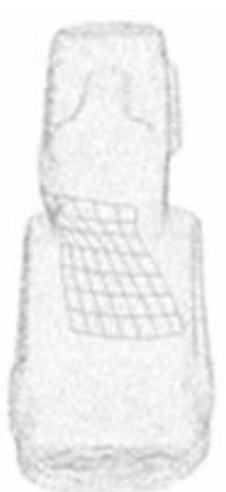

c.

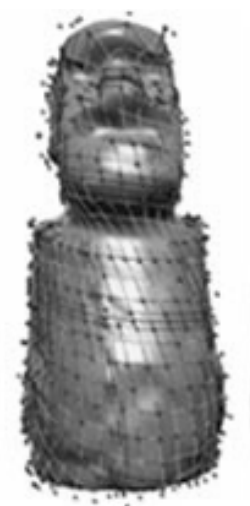

d.

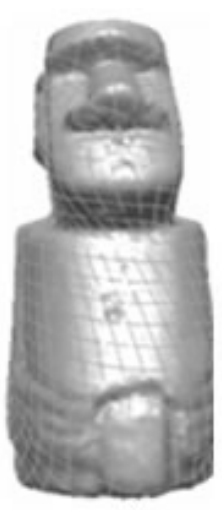

e.

Fig. 8. Proceso de regularización de la malla cuadrilateral. a. Cuadrilaterización previa. b. Proceso de regularización de un cuadrilátero. c. Cuadrilátero regularizado. d. Proceso de regularización de toda la malla cuadrilateral. e. Malla cuadrilateral regularizada.

\section{CONCLUSIONES}

La regularización de una malla cuadrilateral obtenida previamente, se realizó con el fin de generar una rejilla óptima sobre la que pueden trazarse con facilidad parches NURBS, los cuales son una herramienta estándar en los sistemas CAD/CAM y en varias áreas de la computación gráfica y la visión por computador. Tal regularización se realiza mediante curvas geodésicas y B-spline. El esquema del método desarrollado, muestra modelos aceptables aproximados de las superficies de los objetos 3D con topología arbitraria, como se observa en la Fig. 7, conservando los detalles finos de los objetos. Como trabajo futuro, se propone explorar una versión paralela para reducir el costo computacional del método propuesto.

\section{REFERENCIAS}

Boier-Martin, I., H. Rushmeier y J. Jin; Parameterization of triangle meshes over quadrilateral domains. In Proc. Eurographics Symposium on Geometry Processing, 197-207 (2004). 
Bremer, P., H. Edelsbrunner, B. Hamann, y V. Pascucci; A topological hierarchy for functions on triangulated surfaces. TVCG: 10(4), 385-396 (2004).

Cohen-Steiner, D., P. Alliez y M. Desbrun; Variational shape approximation. TOG 23, 3, 905-914. (Proc. SIGGRAPH). (2004).

Dong, S. y otros cuatro autores; Quadrangulating a mesh using laplacian eigenvectors. Technical Report UIUCDCS-R-2005-2583 (2005).

Eck, M. y H. Hoppe; Automatic reconstruction of B-spline surfaces of arbitrary topological type. In Proc. SIGGRAPH, 325-334. (1996).

Friedel, I., P. Schröder y A. Khodakovsky; Variational normal meshes. TOG 23, 4, 1061-1073. (Proc. SIGGRAPH). (2004).

Garland, M., Willmott, A. y Heckbert, P.; Hierarchical face clustering on polygonal surfaces. In Proc. Symp. Interactive 3D Graphics, 49-58,245. (2001).

Hoppe, H.; Progressive meshes. In Proc. SIGGRAPH, 99-108. (1996).

Karni, Z. y C. Gotsman; Spectral compression of mesh geometry. In Proc. SIGGRAPH, 279-286. (2000).

Koren, Y., L. Carmel y D. Harel; ACE:A fast multiscale eigenvectors computation for drawing huge graphs. In Proc. InfoVis '02, 137-144. (2002).

Matthies, G. y F. Schieweck; On the reference mapping for quadrilateral and hexahedral finite elements on multilevel adaptive grids. Computing, Springer-Verlag New York, Inc. Volume 80, Issue 2. Pages: 95-119. ISSN: 0010-485X (2007)

$\mathrm{Ni}, \mathrm{X}$., M. Garland y J. Hart; Fair morse functions for extracting the topological structure of a surface mesh. TOG: 23(3), 613-622 (Proc. SIGGRAPH) (2004).

Schroeder, I., W. Zarge y W. Lorensen; Decimation of triangle meshes. Computer Graphics 26(2), (July), 65-70. (Proc. SIGGRAPH) (1992).

Solín, P. y K. Segeth; Hierarchic higher-order hermite elements on hybrid triangular/quadrilateral meshes. Mathematics and Computers in Simulation. Elsevier Science Publishers B. V. Volume 76, Issue 1-3. Pages 198-204. ISSN: 0378-4754 (2007).

Taubin, G.; Geometrical signal processing on polygonal meshes. In State of the Art Report, Eurographics, 81-96 (2000).

Yvart, A., S. Hahmann y G. Bonneau; Smooth adaptive fitting of $3 D$ models using hierarchical triangular splines. Shape Modelling International (2005). 
